\title{
CT-guided biopsy of the mediastinal masses. Can anatomical relationships predict complications?
}

\author{
Petr Dvorak ${ }^{\mathrm{a}}$, Petr Hoffmann ${ }^{\mathrm{a}}$, Eva Kocova ${ }^{\mathrm{a}}$, Petr Habal ${ }^{\mathrm{b}}$, Marketa Nova ${ }^{\mathrm{c}}$
}

\begin{abstract}
Aim. To retrospectively evaluate the technical features, feasibility, efficacy, accuracy and appropriate complications of CT-guided biopsies of mediastinal masses and correlate them with anatomical relationships in the mediastinum. Methods. Over the course of ten years, 81 percutaneous biopsy procedures in 78 patients for tumors sizes $27-189 \mathrm{~mm}$ in diameter (median length $57.5 \mathrm{~mm}$ ) were performed using only local anesthesia. These interventions were done under the guidance of computed tomography. The patients were men in 30 cases and women in 48 cases, aged 19 to 90 years. Results. In 78 cases (96.3\%) results were either true positive or true negative; in only 3 cases (3.7\%, 3 biopsies of 2 tumors in 2 patients) were histological findings false negative. In $2.3 \%$ of the patients, complications were revealed in the anterior part of the mediastinum, $43.7 \%$ occurred in the middle region, and $14.3 \%$ in the posterior region of the mediastinum. Statistical data analysis verified there was a 3.74 times chance of an increase in complications in the middle mediastinal section in correlation with other anatomical localizations. In only one case was therapeutic intervention for complication necessary. For all other cases, a conservative approach was suitable.

Conclusion. Percutaneous $\mathrm{CT}$ guided biopsies of the mediastinal tumors has a high accuracy rate in establishing the correct diagnosis. Masses in the middle mediastinum are associated with statistically higher incidences of postprocedural complications, which are not serious.
\end{abstract}

Key words: percutaneous biopsy, mediastinal tumors, minimally invasive diagnosis, biopsy complications

Received: May 17, 2018; Revised: July 20, 2019; Accepted: September 14, 2018; Available online: September 24, 2018 https://doi.org/10.5507/bp.2018.058

(c) 2019 The Authors. This is an open access article licensed under the Creative Commons Attribution License (https://creativecommons.org/licenses/by/4.0/).

${ }^{a}$ Department of Radiology, University Hospital, Hradec Kralove, Czech Republic ${ }^{b}$ Department of Cardiac and Thoracic Surgery, University Hospital, Hradec Kralove, Czech Republic 'The Fingerland Department of Pathology, University Hospital, Hradec Kralove, Czech Republic Corresponding author: Petr Hoffmann, e-mail: petr.hoffmann@fnhk.cz

\section{INTRODUCTION}

Mediastinal tumors are uncommon, but they form about $90 \%$ of all mediastinal diseases. The manifestation depends on mechanical pressure on the particular structures in various anatomical parts of the mediastinum. The diseases in the posterior area cause in most cases dysphagia, the middle region cause a high amount of dyspnea, and there is a significant compression of large vascular structures in the anterior area of the mediastinum ${ }^{1}$. The type of neoplasm is characteristic for particular anatomical localization ${ }^{2}$. The type and manifestation also depends on age $\mathrm{e}^{3,4}$.

For establishing the diagnosis of a mediastinal process there are several options. For obtaining tissue samples for histological analysis, the following surgical approaches thoracoscopy (including video-assisted thoracoscopic surgery - VATS), mediastinoscopy, or mediastinotomy can be used. Endoscopic transbronchial or transesophageal techniques with or without ultrasound guidance are other diagnostic possibilities. On the other hand, all these procedures are heavily demanding for the patients, who are not often in a clinically satisfactory status. Percutaneous approaches and detailed imaging guidance can be a more suitable option for the patients while simultaneously aiding with higher diagnostic accuracy.

\section{MATERIALS AND METHODS}

Over the course of ten years, from August 2007 to August 2017, a group of 81 interventional procedures was retrospectively evaluated. This group included 81 biopsies performed on 78 patients. The patients were men in 30 cases $(38.4 \%)$ and women in 48 cases $(61.6 \%)$, aged 19 to 90 years (median 58 years of age). The percutaneous mediastinal biopsies were performed for tumors ranging in sizes of $27-189 \mathrm{~mm}$ in longest diameter (median length 57.5 millimeters). All of these interventional procedures were performed under CT guidance, using the 128-slice CT device Siemens Somatom Definition AS Plus (Siemens, Forchheim, Germany). All the patients were indicated on the basis of the multidisciplinary council (radiologist, pneumologist, thoracic surgeon, and in potentially risky cases also an anesthesiologist). Emphasis was put on patients' clinical status, expected diagnostic benefit, preprocedural preparation, technical aspects of the whole procedure, possible complications, and their prevention and evaluation of the success rate.

Fully informed consent was obtained in all cases by the biopsy performing physician with an explanation of the procedure principles, the disease, possible complications, and their eventual solutions. The procedures were 
performed using Core Semi-Automatic Biopsy System (Bard Magnum Instrument, Bard, Covington, Georgia, USA). In all cases we used a one step approach; the coaxial technique was never performed.

The proper procedure was planned according to a preprocedural imaging examination. The shortest and safest needle distance to reach the lesion was determined. The needle track was planned to also avoid vessels - internal thoracic vessels in the anterior mediastinum, the thoracic aorta and it's branches in the middle mediastinum, and intercostal vessels in the posterior mediastinum. The entry point was defined by placing a skin mark. The distance and biopsy needle angle were measured on the basis of local anesthesia needle short CT imaging. Skin was disinfected and covered with sterile drapes exposing only the entry site. In accordance with the predetermined route, a $16 \mathrm{G} / 18 \mathrm{G}$ of $10 \mathrm{~cm} / 13 \mathrm{~cm} / 16 \mathrm{~cm}$ length with $15 \mathrm{~mm}$ / $22 \mathrm{~mm}$ throw needle was inserted in the proper position into the lesion. The length of the needle was selected according to the skin-lesion distance and the throw was selected on the basis of the biopted process size in the needle direction. The $18 \mathrm{G}$ needle was preferred for insertions affecting pulmonary tissue; in other cases, a 16 $\mathrm{G}$ was suitable. The number of punctures depended on the amount of biopted tissue. The experienced physician performing the biopsy had to be satisfied with it. After obtaining the sample, the needle was carefully removed from the patient's body. The material was put into a sterile $36-38 \%$ formaldehyde solution and the transport system.

All procedures were made using only local anesthesia (Trimecaine, Zentiva, Prague, Czech Republic); conscious sedation was never needed. Parameters of blood coagulation, International Normalized Ratio (INR, lower than 1.5) and activated Partial Thromboplastin Time (aPTT, lower than 1.3) were noted before the biopsy. After the intervention we performed a series of CT scans to exclude the possibility of early complications, such as pneumothorax or hemorrhage. The point of insertion into the skin was cleaned with surgical scrub. The duration of the whole procedure including all preparations never exceeded 30 minutes. The patients were monitored for the remainder of the day by the standard department of pneumology or thoracic surgery. The next morning they were discharged from the hospital after clinical, laboratory, and X-ray examinations.

Only one patient was not discharged from the hospital the next morning. In this case, pneumothorax was revealed after the anterior mediastinal mass biopsy. The patient was short of breath and his clinical status became worse. The chest X-ray examination confirmed progressing pneumothorax and thoracic drainage had to be performed. The chest was drained for four days and the patient stayed in the hospital for a total of five days.

According to the tumor localization in particular mediastinal parts - anterior, middle and posterior - the patients' position on CT table had to be elected. A supine position was suitable for the interventions in the anterior part of mediastinum. For the biopsies in the posterior part of mediastinum, the patients' prone right or left side was appropriately positioned. The approach regarding

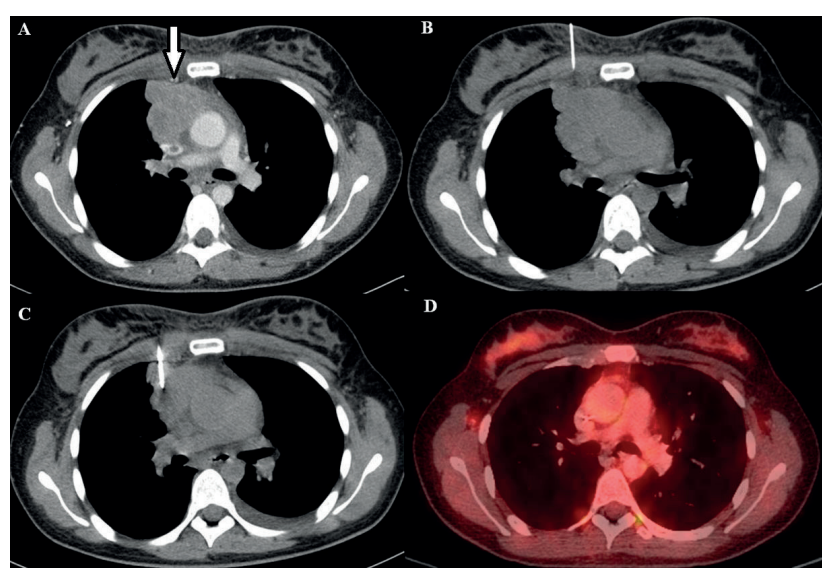

Fig. 1. The biopsy procedure. Vascularized tumor in the right side of anterior mediastinum on contrast medium enhanced CT. (A, internal thoracic vessels are marked with the arrow). The placement of the local anesthesia needle (B) and the biopsy needle ( $\mathrm{C}$, patient in supine position). The histological result was diffuse large B-cell lymphoma (DLBCL). No residual tumorous tissue was revealed on PET/CT after 6 months (D).

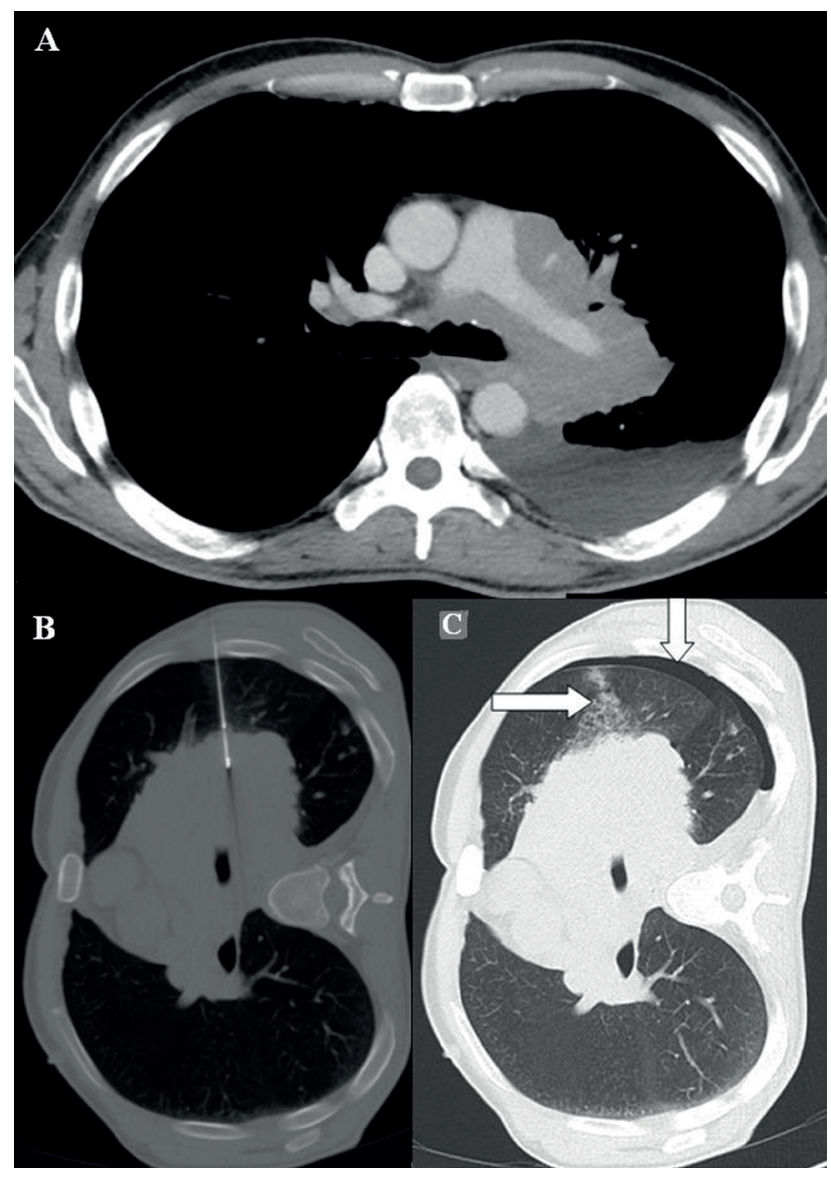

Fig. 2. The biopsy of mass in the middle part of the mediastinum. Vascularized mass containing large mediastinal vessels on contrast medium enhanced CT (A) and the placement of the biopsy needle (B, patient in right side position). CT scan straight after procedure reveals small pneumotorax and pulmonary hemorhage in biopsy needle canal (C, arrows). The biopsy was performed after non-diagnostic transbronchial examination. The $18 \mathrm{G}$ needle with obtaining of one tumorous tissue sample was used. The histological result was mantle cell lymphoma. 
Table 1.

\begin{tabular}{|c|c|c|c|}
\hline \multirow[b]{2}{*}{ Diagnosis } & \multicolumn{3}{|c|}{ Mediastinum } \\
\hline & Anterior & Middle & Posterior \\
\hline DLBCL & 7 & 5 & 6 \\
\hline Hodgkin lymphoma & 8 & 0 & 0 \\
\hline Mantle cell lymphoma & 0 & 2 & 1 \\
\hline Marginal zone lymphoma & 1 & 0 & 0 \\
\hline Follicular lymphoma & 0 & 0 & 1 \\
\hline CLL & 0 & 1 & 1 \\
\hline Plasma cell leukemia & 1 & 0 & 1 \\
\hline Thymoma & 10 & 0 & 0 \\
\hline Thymic carcinoma & 1 & 0 & 0 \\
\hline Thymic T-lymphoma & 2 & 0 & 0 \\
\hline Plasmacytoma & 0 & 0 & 2 \\
\hline IgG4 associated disease & 0 & 0 & 1 \\
\hline Solitary fibrous tumor & 0 & 0 & 1 \\
\hline Synovial sarcoma & 1 & 0 & 0 \\
\hline Myelosarcoma & 0 & 0 & 1 \\
\hline Neurinoma & 0 & 0 & 3 \\
\hline Mixed germinal cell tumor & 1 & 0 & 0 \\
\hline Myxoid chondrosarcoma & 1 & 0 & 0 \\
\hline PNET & 1 & 0 & 0 \\
\hline Metastatic RCC & 0 & 2 & 0 \\
\hline Metastatic breast carcinoma & 1 & 0 & 0 \\
\hline Metastatic NSCLC & 0 & 2 & 0 \\
\hline Dedifferentiated carcinoma & 1 & 0 & 1 \\
\hline No malignant cells verified & 8 & 4 & 2 \\
\hline No. of biopsies & 44 & 16 & 21 \\
\hline
\end{tabular}

DLBCL, Diffuse Large B-Cell Lymphoma CLL, Chronic Lymphocytic Lymphoma PNET, Primitive Neuroectodermal Tumor RCC, Renal Cell Carcinoma

NSCLC, Non Small Cell Lung Carcinoma

Table 2. The incidence of complications in particular parts of the mediastinum.

\begin{tabular}{lccc}
\hline Complication & Anterior & Middle & Posterior \\
\hline Pneumothorax & 0 & 2 & 0 \\
Pulmonary hemorrhage & 0 & 3 & 0 \\
Pulmonary hemorrhage and pneumothorax & 0 & 2 & 1 \\
Drainage & 1 & 0 & 0 \\
None & 43 & 9 & 20 \\
No. of biopsies & 44 & 16 & 21 \\
\hline
\end{tabular}

the middle mediastinal processes was different. In almost every case a percutaneous approach was chosen in the second step in addition to a non-diagnostic endoscopic transbronchial or transesophageal attempt.

Monitored parameters were age, gender, size of the tumor in the longest diameter, localization of the process (anterior, middle and posterior mediastinum), biopsy needle gauge, number of bioptic attempts, known or unknown preprocedural tumorous diseases in the patients' history, complications and their solutions, final histological diagnosis, and correlations with results of selected therapy.

In surgically treated patients, histological results were correlated after the biopsy and after the operation ap- proach. In conservatively treated patients (where the vast majority of diagnoses were lymphomas) were biopsy results correlated with the findings of the bone marrow evaluation and with the results of the targetted treatment. It was considered a success with the permission between histological results of the biopsy and the surgical resection. In the cases where the conservative treatment was selected, it was considered identical to findings of biopsy results and bone marrow evaluations, or to tumorous tissue amounts dissapearing completely on follow-up imaging examinations (Fig. 1). For the following-up we used CT (equal CT device) or PET/CT Discovery VCT 


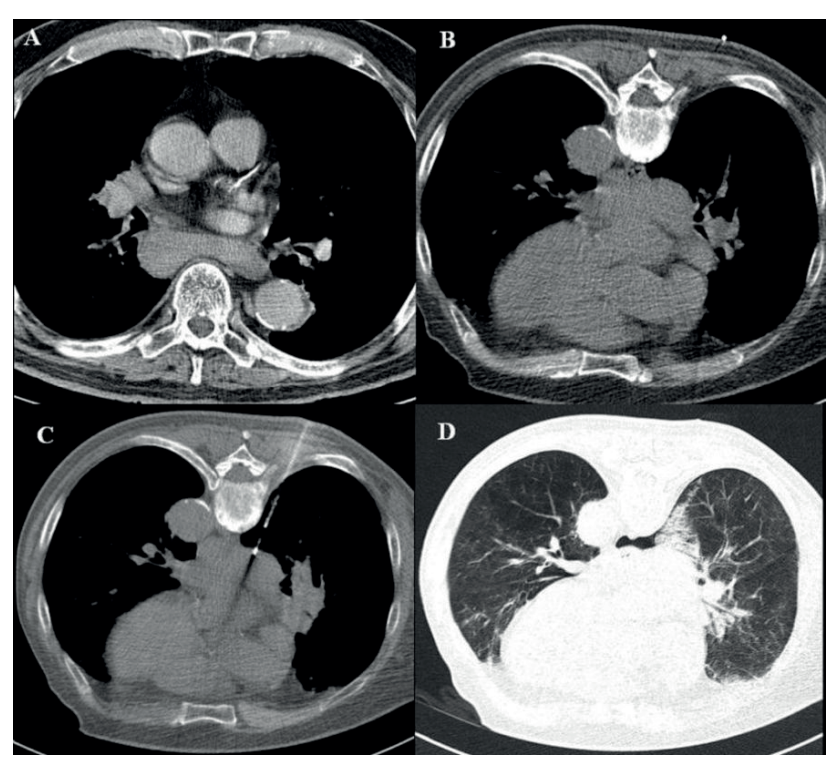

Fig. 3. The biopsy of mass in posterior part of the mediastinum. Vascularized tumor in the prevertebral space in posterior mediastinum (A, patient in the supine position). The placement of the skin mark (B, patient in prone position). The placement of the biopsy needle with the direction affecting pulmonary tissue (C, patient in prone position). Control CT scan straight after procedure revealed small pneumotorax and pulmonary hemorhage in biopsy needle canal (D). The histological result was non-caseating granulomatous inflammatory process and then was sarcoidosis verified.
64 (General Electric Healthcare, Milwaukee, Wisconsin, USA).

If there was a negative histological result and the suspicion of a present tumorous process persisted, the next procedure of the biopsy was performed. The procedure was not much different from the initial intervention; it only focused exactly on the preprocedural localization of the most viable tumorous tissue.

For the basic quantitative statistical evaluation, median and interval data were used. These parameters were correlated with complications, number of bioptic attempts, needle gauge, and localization in the mediastinum using Fisher's exact tests with contingency tables. Qualitative statistical data was descriptively evaluated and quantitative parameters were found using multivariate logistic regression, calculating the Odds ratio. The statistical importance was established at level $P=0.05$. The statistical program NCSS 11 (NCSS, LLC, East Kaysville, Utah, USA) was used.

\section{RESULTS}

A total of 81 interventional procedures where histological results were accessible were included in the study. 81 biopsies were performed in 78 patients. Overall, in only 3 cases ( 3 biopsies of 2 tumors in 2 patients) were histological results false negative $(3.7 \%)$. In 78 cases $(96.3 \%)$ results were true positive or true negative.

The verified diagnoses and the number of cases in particular parts of the mediastinum are in Table 1 . The $16 \mathrm{G}$ needle was utilized in 58 biopsies (71.6\%) and 18

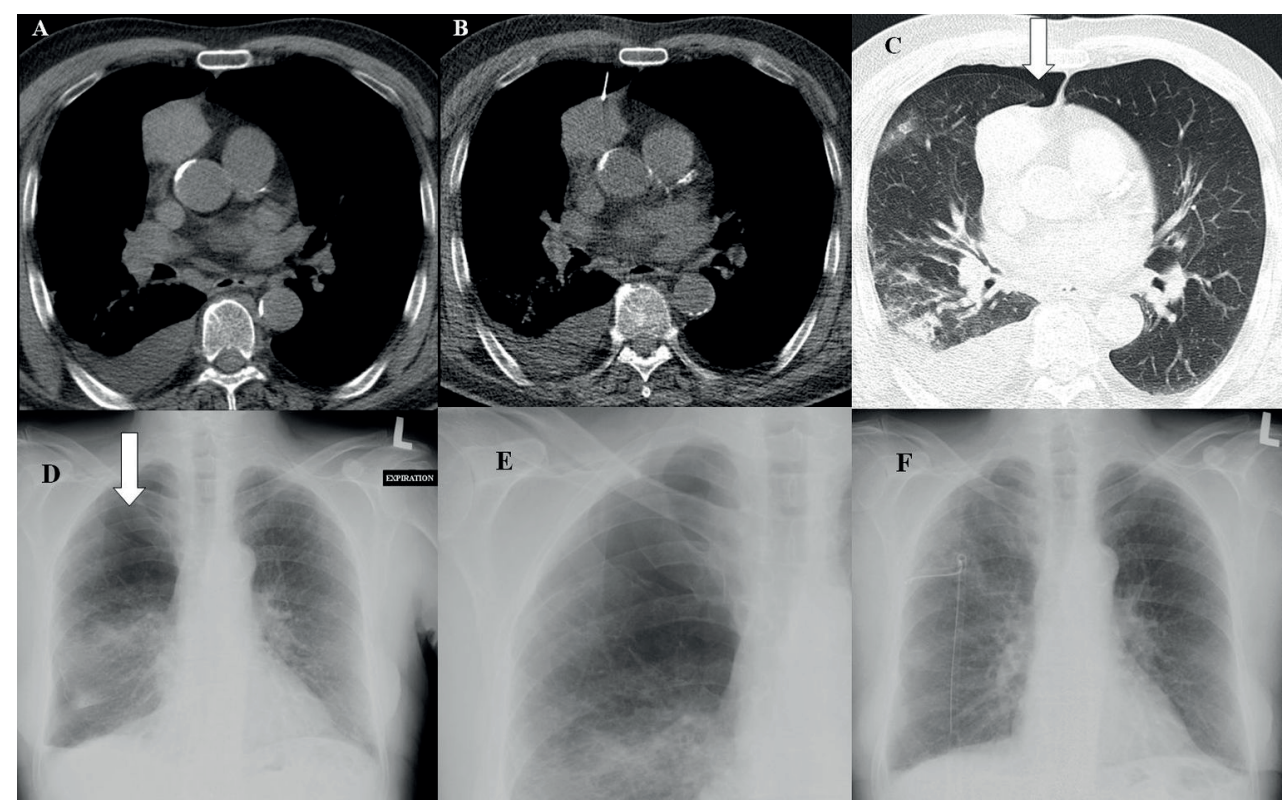

Fig. 4. The biopsy of mass in anterior part of the mediastinum. The bordered process on preprocedural unenhanced CT examination (A) and the placement of the biopsy needle (B, patient in supine position). Control CT scan straight after procedure revealed small pneumotorax ( $\mathrm{C}$, arrow). The control $\mathrm{X}$ ray examination 3 hours after the biopsy revealed progressing pneumotorax $(\mathrm{D} ; \mathrm{E}$ in detailed view). The chest drainage was necessary (F). The drainage for pneumotorax required the patient to stay in the hospital for 4 days. The histological result was thymoma. 
G needle in 23 interventions (28.4\%). The complications were revealed in 7 cases using the $16 \mathrm{G}$ needle $(7 / 58$; $12.07 \%$ ) and in 2 interventions using the $18 \mathrm{G}$ needle $(2 / 23 ; 8.69 \%)$. The Fisher's exact test proved statistically non-significant relationship between needle gauge and complication rate $(P=0.80608)$.

Th number of bioptic attempts was variable. One sampling in 11 cases (13.6\%), two samplings in 54 interventions (66.7\%), three samplings in 13 biopsies ( $16.1 \%)$ and four samplings in 3 cases $(3.6 \%)$ were performed. One attempt was determined for interventions affecting the lungs of patients with high risk of complications. Two biopsies in this group were complicated with pneumothorax; in one case, the drainage was necessary. Two punctures were sufficient in the majority of interventions. In seven cases $(7 / 54 ; 12.9 \%)$ complications were revealed, but an interventional approach was never required. Three and four bioptic attempts were optimal for large masses with an emphasis on targetting various parts of processes. The complication was never revealed in this group. The Fisher's exact test did not prove statistically significant relationship between the number of punctures and complication rate $(P=0.30212)$.

False negative results were confirmed in two patients. In one case a biopsy was repeated and the histological results showed complete necrotic tissue in both attempts. The patient underwent an operation and the final result confirmed low grade neurofibrosarcoma with necrotic components. The biopsy technique was standard, but the samples were obtained from the necrotic parts of the mass. In the second case of the anterior mediastinal mass biopsy, the histological result showed fatty tissue with fibrotic parts; no malignant cells were verified. Subsequently, mediastinoscopy was performed and Hodgkin's lymphoma was confirmed.

All true positive results were considered as the final diagnosis and deemed sufficient for treatment. Lymphomas and leukemias were treated by hematooncologists with targetted systemic medication. The verification of disseminated tumorous diseases was enough for the oncologists and their treatment. Other diagnoses (thymic tumors and neurinomas) were treated surgically in the thoracic department of our hospital. The histological results of resected tumors and the biopsies were compared. In systemic treatments (lymphomas) the success was intensively observed within the follow-up examinations.

In the true negative results, a long-term follow-up was performed. In the anterior part of the mediastinum the benign results showed ectopic thyroid tissue in two cases and fibrous scar-like tissue in four cases. All these patients had known malignant diseases marked in their clinical history (DLBCL in three cases and Hodgkin's lymphoma in one case). Their follow-up examinations verified a significant amount decrease in a period of more than three years after the intervention. In one case diagnosis was confirmed by surgical resection. In the middle part of the mediastinum, extramedullary hematopoiesis was diagnosed in one case and fibrotic tissue in a second case. This diagnosis was confirmed by an autopsy. In the posterior part of the mediastinum, a granulomatous inflam- matory process and sarcoidosis were verified in one case and fibroproductive pleuritis was diagnosed in another.

The complications of the biopsies were observed and divided into groups: pneumotorax, pulmonary hemorrhage, both pulmonary hemorrhage and pneumotorax, pneumotorax requiring drainage, and none, and correlated with the number of bioptic attempts, needle gauge, and anatomical part of the mediastinum (Table 2). However, the anatomical mass localization in the middle part of the mediastinum has a statistically significant higher incidence of complications.

In the anterior part of the mediastinum complications were revealed in only one case of 44 interventions $(2.3 \%)$, 7 cases of 16 biopsies in the middle area (43.7\%), and in the posterior area 3 cases of 21 interventions (14.3\%) (Fig. 2 and 3). The multivariate logistic regression determined the Odds ratio to be 3.74 . The complications of the biopsies in this group occur 3.74 times more often in the middle mediastinal part in correlation with other anatomical localizations. Nevertheless, in only one case was therapeutic intervention - chest drainage - necessary (Fig. 4). This drainage for pneumothorax required the patient to stay in the hospital for 4 days, with an additional fifth day for recovery. For other complications a conservative approach was suitable.

\section{DISCUSSION}

To obtain a relevant tumorous sample from the soft tissue mediastinal mass we can use different diagnostic approaches. All these techniques have clear advantages, but there are also limitations. The goal was to present the experiences and results as well as find a role for the percutaneous approach using CT-guidance and only local anesthesia. A correct combination of diagnostic methods should be used in different situations for establishing the final diagnosis.

The pathological tumorous process stays in the mediastinum, which is the localization of various medical specializations: pneumology, gastroenterology, thoracic surgery, and in addition to all cases, imaging methods. All specialties use their own methods and guidance - endobronchial ultrasonography (EBUS), transesophageal biopsy, or surgical approaches (mediastinoscopy, mediastinotomy, and thoracoscopy including VATS). The limitations are clear. The transesophageal approach (EUS-FNA, endoscopic ultrasound - fine needle aspiration) can reach diseases of the posterior mediastinum, including the lower and left paratracheal localization of the middle mediastinum ${ }^{5}$. The transbronchial approach (EBUS-TBNA, endobronchial ultrasound - transbronchial needle aspiration) can reach pretracheal and left paratracheal spaces in the middle mediastinum. These types of intervention are used in the staging diagnosis of lung cancer, but are not widely used in the primary mediastinal mass assessment ${ }^{6}$. Endoscopic and transbronchial techniques are also demanding and should be performed by an experienced physician; general anesthesia is necessary. The procedure is challenging for the patients and the quality any of obtained tissue samples 
depend on the needle gauge. The obtained material is thin or fragmented, because of the aspiration technique. The diagnostic sensitivity of endoscopic and transbronchial interventions varies around $80 \%\left(\right.$ ref. $\left.^{7}\right)$. The combination of these approaches can increase the success rate $^{8}$. The majority of studies are dedicated to lung cancer staging. The study of EBUS guided mediastinal lymphoma diagnosis and subtyping revealed the correct diagnosis in $67 \%$ with de novo lymphoma and $81 \%$ of patients with relapsed lymphoma ${ }^{9}$. The true advantage of endoscopic or transbronchial interventions is the low complication rate, but possible inconveniences can be serious ${ }^{10,11}$.

The advantages of the surgical approaches are obvious. The procedure uses a direct approach to the mediastinum, obtaining a sample is visibly controlled, and the patient's cooperation is optimal due to general anesthesia. On the other hand, the procedure is a proper operating performance, and thus contain appropriate risks and complications. Among presented diagnostic methods they are mostly challenging for the patients. The mediastinoscopy can reach upper retrosternal space, the trachea, and surrounding and subcarinal regions using the cervical approach $^{12}$. Modifications such as parasternal mediastinoscopy, or using subxiphoid approach are performed in particular cases. The thoracoscopy (including VATS) is a diagnostic possibility for the pleural cavity diseases. This procedure can be used in some indicated cases ${ }^{13}$. The complications of surgical modalities are not common, but they are serious ${ }^{14-16}$.

Histological verification of all mediastinal masses is important for the selection of appropriate therapy. Establishing the final diagnosis should be fast and uncomplicated. The eventual delay due to diagnostic complications could cause tumorous process progression in a relatively short time with a steadily worsening prognosis.

The published results in literature are slightly different. The overall diagnostic accuracy varies from $83.6 \%$ to $97.4 \%$. For lymphoma, diagnostic accuracy results are similar, but for lymphoma subtyping outcomes are less reliable ${ }^{17-21}$. The complication rate was low in all studies, varrying from $3.85 \%$ to $7 \%$ (ref. ${ }^{17-21}$ ). The vast majority were small pneumothoraces without need of intervention or hospitalization ${ }^{19}$. These results are in concordance with published studies. The diagnostic accuracy of our results $(96.3 \%)$ is in the upper part of the before-mentioned interval. The overall complication rate $(13.7 \%)$ is higher than published data, but excluding middle mediastinum mass biopsies, the complication is rate comparable $(6.06 \%)$. Our access is invasive to aggressive, with excellent diagnostic accuracy and yield. Although there is a higher complication rate, it is caused due to interventions in the middle part of mediastinum.

\section{CONCLUSION}

Percutaneous CT-guided biopsy of the mediastinal masses has a substantially high diagnostic accuracy in establishing final diagnosis. The procedure's results are sufficient for all specialties' treatment. The intervention can reach all mediastinal parts using only local anesthesia. The complication rate is noticeably low, excluding middle mediastinal masses. The biopsies in the middle mediastinum are associated with statistically higher incidences of postprocedural complications, which are not serious.

Acknowledgement: The authors are grateful to Dr. Olga Cermakova for considerable help with statistical data analysis and to Jennifer Raak BA for language correction of the manuscipt.

This work was supported by MH CZ - DRO (UHHK, 00179906), by MH CZ NT13531-3/2013 and by SVV 260398/2017.

Author contributions: PD, PHo: biopsies performing, manuscript writing, final approval; PHo, EK: literature search, data analysis; PHa: following-up, data analysis; MN: histological verification, final approval.

Conflict of interest statement: None declared.

\section{REFERENCES}

1. Dähnert W. Radiology Review Manual, 7th ed. Philadelphia, Lippincott Williams \& Wilkins, 1264 pp., 2011.

2. Juanpere S, Cañete N, Ortuño P, Martínez S, Sanchez G, Bernado L. A Diagnostic Approach to the Mediastinal Masses. Insights Imaging 2013;4(1):29-52.

3. Pearson JK, Tan GM. Pediatric Anterior Mediastinal Mass: A Review Article. Semin Cardiothorac Vasc Anesth 2015;19(3):248-54.

4. Liu T, Al-Kzayer LFY, Xie X, Fan H, Sarsam SN, Nakazawa Y, Chen L. Mediastinal lesions across the age spectrum: a clinicopathological comparison between pediatric and adult patients. Oncotarget 2017;8(35):59845-53.

5. Mondoni M, D'Adda A, Terraneo S, Carlucci P, Radovanovic D, Di Marco F, Santus P. Choose the best route: ultrasound-guided transbronchial and transesophageal needle aspiration with echobronchoscope in the diagnosis of mediastinal and pulmonary lesions. Minerva Med 2015;106(5 Suppl 1):13-9.

6. Kużdżał J, Szlubowski A. Ultrasound-guided transbronchial and transesophageal needle biopsy in the mediastinal staging of lung cancer. Thorac Surg Clin 2012;22(2):191-203.

7. Dhooria S, Aggarwal AN, Gupta D, Behera D, Agarwal R. Utility and Safety of Endoscopic Ultrasound With Bronchoscope-Guided FineNeedle Aspiration in Mediastinal Lymph Node Sampling: Systematic Review and Meta-Analysis. Respir Care 2015;60(7):1040-50.

8. Badaoui A, Dahlqvist C, Rahier J, Weynand B, Ocak S, Deprez P, Eucher $P$, Duplaquet F. Combined endoscopic ultrasonography and endobronchial ultrasound-fine-needle aspiration for evaluation of mediastinal lymph nodes. Endosc Ultrasound 2014;3(3 Suppl 1):S9.

9. Grosu HB, Iliesiu M, Caraway NP, Medeiros LJ, Lei X, Jimenez CA Morice RC, Casal RF, Ost D, Eapen GA. Endobronchial UltrasoundGuided Transbronchial Needle Aspiration for the Diagnosis and Subtyping of Lymphoma. Ann Am Thorac Soc 2015;12(9):1336-44.

10. Ceuterick V, Decaluwé $H$, Coosemans W, Nafteux $P$, Van Veer $H$, Van Raemdonck D, De Leyn P. Esophageal wall rupture after EUSFNA for mediastinal staging: report of two cases. Acta Chir Belg 2016;116(1):48-50

11. Çağlayan B, Yılmaz A, Bilaçeroğlu S, Cömert SŞ, Demirci NY, Salepçi B. Complications of Convex-Probe Endobronchial Ultrasound-Guided Transbronchial Needle Aspiration: A Multi-Center Retrospective Study. Respir Care 2016;61(2):243-8.

12. Onat S, Ates G, Avcı A, Yıldız T, Birak A, Akgul Ozmen C, Ulku R. The role of mediastinoscopy in the diagnosis of non-lung cancer diseases. Ther Clin Risk Manag 2017;13:939-43.

13. Habal P, Simek J. Videothoracoscopy, standard and uncommon indications. Rozhl Chir 2007;86(8):442-7.

14. Bilgin Büyükkarabacak $Y$, Taslak Şengül $A$, Meydan BC, Çelik $B$, Pirzirenli MG, Gürz S, Sürücü ZP, Başoğlu A. The risk of tumor cell 
dissemination in mediastinoscopy: a cytological study. Turk J Med Sci 2015;45(4):872-6.

15. Elsayed H. Haemothorax after mediastinoscopy: a word of caution. Eur J Cardiothorac Surg 2012;41(1):138-9.

16. Billè $A$, Longman $C$, Pilling J. Aortic pseudoaneurysm: a rare complication of mediastinoscopy. Gen Thorac Cardiovasc Surg 2013;61(3):160-2.

17. Priola AM, Priola SM, Cataldi A, Ferrero B, Garofalo G, Errico L, Marci V, Fava C. CT-guided percutaneous transthoracic biopsy in the diagnosis of mediastinal masses: evaluation of 73 procedures. Radiol Med 2008;113(1):3-15.

18. Petranovic M, Gilman MD, Muniappan A, Hasserjian RP, Digumarthy SR, Muse VV, Sharma A, Shepard JA, Wu CC. Diagnostic Yield of CT-
Guided Percutaneous Transthoracic Needle Biopsy for Diagnosis of Anterior Mediastinal Masses. AJR Am J Roentgenol 2015;205(4):774-9.

19. de Margerie-Mellon $C$, de Bazelaire $C$, Amorim S, Brice $P$, Tazi $A$, Brière J, Frija J, de Kerviler E. Diagnostic Yield and Safety of Computed Tomography-guided Mediastinal Core Needle Biopsies. J Thorac Imaging 2015;30(5):319-27.

20. Kulkarni S, Kulkarni A, Roy D, Thakur MH. Percutaneous computed tomography-guided core biopsy for the diagnosis of mediastinal masses. Ann Thorac Med 2008;3(1):13-7.

21. Kim H, Park CM, Lee SM, Goo JM. C-Arm Cone-Beam CT Virtual Navigation-Guided Percutaneous Mediastinal Mass Biopsy: Diagnostic Accuracy and Complications. Eur Radiol 2015;25(12):3508-17. 